Article

\title{
Chemical Compositions, Mosquito Larvicidal and Antimicrobial Activities of Essential Oils from Five Species of Cinnamomum Growing Wild in North Central Vietnam
}

\author{
Do N. Dai ${ }^{1,2, *}$, Nguyen T. Chung ${ }^{1}$, Le T. Huong ${ }^{3}$, Nguyen H. Hung ${ }^{4} \oplus$, Dao T.M. Chau ${ }^{5}$, \\ Nguyen T. Yen ${ }^{3}$ and William N. Setzer $6,7, *$ (D) \\ 1 Graduate University of Science and Technology, Vietnam Academy of Science and Technology, \\ 18-Hoang Quoc Viet, Cau Giay, Hanoi 10072, Vietnam; chungpuhoat@gmail.com \\ 2 Faculty of Agriculture, Forestry and Fishery, Nghe An College of Economics, 51-Ly Tu Trong, Vinh City 4300, \\ Nghe An Province, Vietnam \\ 3 School of Natural Science Education, Vinh University, 182 Le Duan, Vinh City 4300, Nghệ An Province, \\ Vietnam; lehuong223@gmail.com (L.T.H.); nguyenthiyenth92@gmail.com (N.T.Y.) \\ 4 Center for Advanced Chemistry, Institute of Research and Development, Duy Tan University, \\ 03 Quang Trung, Da Nang 5000, Vietnam; nguyenhuyhung@duytan.edu.vn \\ 5 Institute of Environmental Biochemistry, Vinh University, 182 Le Duan, Vinh City 4300, Nghệ An Province, \\ Vietnam; daochau27@gmail.com \\ 6 Aromatic Plant Research Center, 230 N 1200 E, Suite 100, Lehi, UT 84043, USA \\ 7 Department of Chemistry, University of Alabama in Huntsville, Huntsville, AL 35899, USA \\ * Correspondence: daidn23@gmail.com (D.N.D.); wsetzer@chemistry.uah.edu (W.N.S.)
}

Academic Editor: Giovanni Benelli

Received: 20 January 2020; Accepted: 17 February 2020; Published: 12 March 2020

\begin{abstract}
Members of the genus Cinnamomum (Lauraceae) have aromatic volatiles in their leaves and bark and some species are commercially important herbs and spices. In this work, the essential oils from five species of Cinnamomum (C. damhaensis, C. longipetiolatum, C. ovatum, C. polyadelphum and C. tonkinense) growing wild in north central Vietnam were obtained by hydrodistillation, analyzed by gas chromatography and screened for antimicrobial and mosquito larvicidal activity. The leaf essential oil of C. tonkinense, rich in $\beta$-phellandrene (23.1\%) and linalool (32.2\%), showed excellent antimicrobial activity (MIC of $32 \mu \mathrm{g} / \mathrm{mL}$ against Enterococcus faecalis and Candida albicans) and larvicidal activity ( $24 \mathrm{~h} \mathrm{LC} 50$ of $17.4 \mu \mathrm{g} / \mathrm{mL}$ on Aedes aegypti and $14.1 \mu \mathrm{g} / \mathrm{mL}$ against Culex quinquefasciatus). Cinnamomum polyadelphum leaf essential oil also showed notable antimicrobial activity against Gram-positive bacteria and mosquito larvicidal activity, attributable to relatively high concentrations of neral (11.7\%) and geranial (16.6\%). Thus, members of the genus Cinnamomum from Vietnam have shown promise as antimicrobial agents and as potential vector control agents for mosquitoes.
\end{abstract}

Keywords: Lauraceae; Aedes aegypti; Aedes albopictus; Culex quinquefasciatus; antibacterial; antifungal

\section{Introduction}

The Lauraceae is a large family of tropical and subtropical trees and shrubs [1]. In this family, the genus Cinnamomum is comprised of around 250 species with concentrations in east and southeast Asia [1]. Vietnam is home to 45 species of Cinnamomum [2,3], many of which are used in traditional medicine, for essential oils, as well as for timber $[4,5]$.

We are in the midst of a post-antibiotic era. Numerous pathogenic microorganisms have developed resistance to commonly used antibiotic agents [6,7]. For example, Klebsiella pneumoniae [8], Pseudomonas aeruginosa [9] and Staphylococcus aureus [10], three organisms that are major causes of nosocomial 
infections, have developed extremely drug resistant (XDR) strains. Likewise, antibiotic resistance is increasing in fungi such as Candida ssp. and Aspergillus spp. [11]. Essential oils have shown promise as complementary or adjuvant therapies for combating antimicrobial resistance [12-19].

Mosquitoes have been and continue to be the deadliest animals on earth. Aedes aegypti (L.) (Diptera: Culicidae) and Ae. albopictus (Skuse) are vectors for the arboviral diseases dengue, Zika, chikungunya and yellow fever and Ae. aegypti is also a vector for the emerging Rift Valley fever virus [20]. Culex quinquefasciatus (Say) is a vector of West Nile virus, Saint Louis encephalitis virus and lymphatic filariasis [21]. Culex quinquefasciatus may also serve as a vector in emerging viral diseases such as Zika virus [22], Sindbis virus [23] and Usutu virus [24]. Unfortunately, insecticidal resistance of these mosquito species is increasing leading to failure of vector control programs in many locations [25]. Furthermore, populations of Ae. aegypti [26], Ae. albopictus [27] and Cx. quinquefasciatus [28] are showing widespread resistance to commonly used larvicidal agents. It has been suggested that essential oils may serve as alternative and more ecologically benign mosquito larvicidal agents [29-31].

Because of the biological activities and traditional uses of members of the Cinnamomum genus, we hypothesize that Cinnamomum species from Vietnam may also exhibit potentially useful biological activities. As part of our ongoing investigations into the essential oils of Vietnamese Cinnamomum [32-34], we have obtained, analyzed and carried out antimicrobial and larvicidal screening of Cinnamomum ovatum C.K. Allen (syn. Cinnamomum rigidissimum H.T. Chang), Cinnamomum tonkinense (Lecomte) A. Chev. (syn. Cinnamomum albiflorum var. tonkinense Lecomte), Cinnamomum damhaensis Kosterm., Cinnamomum longipetiolatum H.W. Li and Cinnamomum polyadelphum (Lour.) Kosterm. (syn. Laurus polyadelpha Lour., Cinnamomum litseafolium Lecomte, Cinnamomum litseafolium var. denticupulatum Liou, Cinnamomum saigonicum Farw, Camphorina saigonica Farw).

\section{Results}

\subsection{Essential Oil Collection and Analysis}

Plant materials were collected from mature Cinnamomum trees from different locations in north central Vietnam. The collection details and essential oil yields of the Cinnamomum species are summarized in Table 1. The essential oils were analyzed by gas-chromatography-mass spectrometry (GC-MS) and gas chromatography-flame ionization detector (GC-FID). The chemical compositions of the Cinnamomum species are presented in Table 2.

Table 1. Collection details for Cinnamomum species from north central Vietnam.

\begin{tabular}{|c|c|c|c|c|c|c|}
\hline $\begin{array}{l}\text { Cinnamomum } \\
\text { Species }\end{array}$ & $\begin{array}{l}\text { Vietnamese } \\
\text { Name }\end{array}$ & $\begin{array}{l}\text { Voucher } \\
\text { Numbers }\end{array}$ & Part & $\begin{array}{l}\text { Yield, } \\
\% \text { v/w }\end{array}$ & $\begin{array}{l}\text { Collection } \\
\text { Month/Year }\end{array}$ & Collection Location \\
\hline $\begin{array}{l}\text { Cinnamomum } \\
\text { ovatum }\end{array}$ & Re trứng & DND-762 & $\begin{array}{l}\text { Leaf } \\
\text { Stems }\end{array}$ & $\begin{array}{l}0.60 \\
0.21\end{array}$ & April/2019 & $\begin{array}{l}\text { Chau Hoan Commune, Pù Huống } \\
\text { Nature Reserve } \\
19^{\circ} 28^{\prime} 12^{\prime \prime} \mathrm{N}, 104^{\circ} 56^{\prime} 45^{\prime \prime} \text {, elev. } 374 \mathrm{~m}\end{array}$ \\
\hline $\begin{array}{l}\text { Cinnamomum } \\
\text { tonkinense }\end{array}$ & $\begin{array}{c}\text { Re xanh, Re bắc, } \\
\text { Quế bắc }\end{array}$ & DND-768 & Leaf & 0.33 & April/2019 & $\begin{array}{l}\text { Chau Hoan Commune, Pù Huống } \\
\text { Nature Reserve } \\
19^{\circ} 28^{\prime} 12^{\prime \prime} \mathrm{N}, 104^{\circ} 56^{\prime} 45^{\prime \prime} \text {, elev. } 374 \mathrm{~m}\end{array}$ \\
\hline $\begin{array}{l}\text { Cinnamomum } \\
\text { damhaensis }\end{array}$ & Re đầm hà & DND-786 & Leaf & 0.30 & July/2019 & $\begin{array}{l}\text { Huong Phu Commune, Nam Đông } \\
\text { District, Bach Ma National Park } \\
16^{\circ} 12^{\prime} 47^{\prime \prime} \mathrm{N}, 107^{\circ} 43^{\prime} 33^{\prime \prime} \text {, elev. } 101 \mathrm{~m}\end{array}$ \\
\hline $\begin{array}{l}\text { Cinnamomum } \\
\text { longipetiolatum }\end{array}$ & Re cuống dài & DND-800 & Leaf & 1.35 & August/2019 & $\begin{array}{l}\text { Nam Nhong Commune, Que Phong } \\
\text { District, Pù Hoạt Nature Reserve } \\
19^{\circ} 30^{\prime} 24^{\prime \prime} \mathrm{N}, 104^{\circ} 42^{\prime} 52^{\prime \prime} \mathrm{E} \text {, elev. } 667 \mathrm{~m}\end{array}$ \\
\hline $\begin{array}{l}\text { Cinnamomum } \\
\text { polyadelphum }\end{array}$ & $\begin{array}{l}\text { Quế bời lời, Miếng } \\
\text { sành, Tà Dúi, Ô } \\
\text { dược, Đam dao, } \\
\text { Hậu phát }\end{array}$ & DND-813 & Leaf & 1.20 & August/2019 & $\begin{array}{l}\text { Nam Nhong Commune, Que Phong } \\
\text { District, Pù Hoạt Nature Reserve } \\
19^{\circ} 30^{\prime} 24^{\prime \prime} \mathrm{N}, 104^{\circ} 42^{\prime} 52^{\prime \prime} \mathrm{E} \text {, elev. } 667 \mathrm{~m}\end{array}$ \\
\hline
\end{tabular}


Table 2. Chemical compositions (\%) of Cinnamomum essential oils from north central Vietnam.

\begin{tabular}{|c|c|c|c|c|c|c|c|c|c|}
\hline $\mathbf{N}^{\circ}$ & Compounds & $\mathrm{RI}^{\mathrm{a}}$ & $\mathbf{R I}^{\mathrm{b}}$ & C.ov. ${ }^{\mathrm{c}}$ & C.ov. ${ }^{\mathrm{c}}$ & C. to. ${ }^{\mathrm{d}}$ & C. $d a .^{\mathrm{e}}$ & C. lo..$^{\mathrm{f}}$ & C.po.g \\
\hline & & & & Leaf & Stem & Leaf & Leaf & Leaf & Leaf \\
\hline 1 & $\alpha$-Thujene & 930 & 924 & - & - & 0.4 & - & - & 0.3 \\
\hline 2 & $\alpha$-Pinene & 939 & 932 & 2.1 & 1.6 & 4 & 0.3 & 2.9 & 4.3 \\
\hline 3 & $\alpha$-Fenchene & 953 & 945 & - & - & - & 0.1 & - & 0.2 \\
\hline 4 & Camphene & 955 & 946 & 0.7 & 0.6 & 0.4 & 0.2 & 0.3 & 1.9 \\
\hline 5 & Sabinene & 978 & 969 & - & - & 3.4 & 0.7 & 0.5 & 0.4 \\
\hline 6 & $\beta$-Pinene & 984 & 974 & 0.9 & 0.6 & 2.1 & 0.2 & 1.7 & 2.4 \\
\hline 7 & Myrcene & 992 & 988 & 0.2 & 0.1 & 3.1 & 0.1 & 0.2 & 2.1 \\
\hline 8 & Dehydroxy-trans-linalool oxide & 995 & 991 & - & - & - & - & 0.7 & - \\
\hline 9 & Dehydroxy-cis-linalool oxide & 1008 & 1006 & - & - & - & - & 0.6 & - \\
\hline 10 & $\alpha$-Phellandrene & 1010 & 1002 & 1.3 & 0.3 & 4.8 & - & - & 0.2 \\
\hline 11 & $\alpha$-Terpinene & 1022 & 1014 & - & - & 0.4 & - & 0.2 & \\
\hline 12 & p-Cymene & 1030 & 1020 & 0.7 & 0.4 & 0.5 & 0.7 & 0.2 & 0.7 \\
\hline 13 & Limonene & 1035 & 1024 & 0.9 & 0.8 & 3.4 & 0.2 & 0.3 & 5.4 \\
\hline 14 & $\beta$-Phellandrene & 1036 & 1025 & - & - & 23.1 & - & - & - \\
\hline 15 & 1,8-Cineole & 1038 & 1026 & 0.2 & 0.6 & 9.8 & 1 & 2.5 & 0.8 \\
\hline 16 & (E)-ß-Ocimene & 1049 & 1044 & 0.3 & 0.2 & 0.3 & - & 0.8 & - \\
\hline 17 & $\gamma$-Terpinene & 1063 & 1054 & - & - & 0.5 & - & 0.3 & - \\
\hline 18 & Terpinolene & 1094 & 1086 & 0.2 & 0.1 & 0.2 & - & - & - \\
\hline 19 & Rosefuran & 1098 & 1091 & - & - & - & - & - & 0.1 \\
\hline 20 & Perillene & 1104 & 1102 & - & - & - & - & - & 0.2 \\
\hline 21 & Linalool & 1105 & 1095 & 5.9 & 8.3 & 32.2 & 44.8 & 75.7 & 3.2 \\
\hline 22 & Hotrienol & 1107 & 1104 & - & - & - & - & 3.2 & - \\
\hline 23 & Isocitral & 1147 & 1140 & - & - & - & - & - & 0.2 \\
\hline 24 & Camphor & 1156 & 1141 & - & - & - & - & - & 32.2 \\
\hline 25 & Nerol oxide & 1158 & 1154 & - & - & - & - & 0.2 & - \\
\hline 26 & Isoneral & 1166 & 1162 & - & - & - & - & - & 0.6 \\
\hline 27 & cis-Linalool oxide (pyranoid) & 1174 & 1170 & - & - & - & - & 3.2 & - \\
\hline 28 & trans-Linalool oxide (pyranoid) & 1177 & 1173 & - & - & - & - & 2.7 & - \\
\hline 29 & Borneol & 1178 & 1165 & - & - & 0.2 & - & - & 1.6 \\
\hline 30 & Isogeranial & 1184 & 1180 & - & - & - & - & - & 0.9 \\
\hline 31 & Terpinen-4-ol & 1187 & 1174 & - & - & 0.7 & 0.4 & 0.5 & 0.4 \\
\hline 32 & $\alpha$-Terpineol & 1200 & 1186 & - & - & 1.7 & 0.2 & 0.6 & 0.6 \\
\hline 33 & Decanal & 1208 & 1201 & - & - & 0.2 & - & - & 0.2 \\
\hline 34 & Citronellol & 1228 & 1223 & - & - & - & - & - & 0.4 \\
\hline 35 & Nerol & 1231 & 1227 & - & - & - & - & - & 0.8 \\
\hline 36 & Cuminal & 1238 & 1238 & - & - & - & 0.1 & - & - \\
\hline 37 & Neral & 1245 & 1235 & - & - & - & - & - & 11.7 \\
\hline 38 & Geraniol & 1255 & 1249 & - & - & - & - & 0.2 & 1.9 \\
\hline 39 & Geranial & 1274 & 1264 & - & - & - & - & - & 16.6 \\
\hline 40 & (E)-Cinnamaldehyde & 1278 & 1267 & - & - & - & - & - & 0.2 \\
\hline 41 & Bornyl acetate & 1294 & 1287 & 0.5 & 0.5 & 0.1 & - & - & 0.1 \\
\hline 42 & Safrole & 1299 & 1285 & - & 0.2 & - & - & - & - \\
\hline 43 & $\delta$-Elemene & 1348 & 1335 & - & - & 0.2 & - & - & - \\
\hline 44 & Eugenol & 1367 & 1356 & 70.5 & 71.2 & 0.2 & - & - & 0.4 \\
\hline 45 & $\alpha$-Ylangene & 1385 & 1373 & 0.4 & 0.3 & - & - & - & - \\
\hline 46 & $\alpha$-Copaene & 1389 & 1374 & - & - & 0.4 & - & - & 0.1 \\
\hline 47 & $\beta$-Elemene & 1403 & 1389 & - & - & 0.3 & 0.7 & - & - \\
\hline 48 & Methyl eugenol & 1409 & 1403 & - & 0.3 & - & 0.1 & - & 0.3 \\
\hline 49 & $\beta$-Caryophyllene & 1437 & 1417 & 1.9 & 1 & 1.8 & 0.2 & - & 1.3 \\
\hline 50 & trans- $\alpha$-Bergamotene & 1445 & 1432 & 0.2 & 0.2 & 0.1 & - & - & - \\
\hline 51 & allo-Aromadendrene & 1457 & 1458 & 0.1 & - & - & 0.3 & - & - \\
\hline 52 & $\alpha$-Humulene & 1471 & 1452 & 0.3 & - & 0.6 & - & - & 0.2 \\
\hline 53 & $\alpha$-Amorphene & 1483 & 1483 & - & - & - & 0.3 & - & - \\
\hline 54 & $\beta$-Selinene & 1489 & 1489 & - & - & - & 19.1 & - & - \\
\hline 55 & trans- $\beta$-Bergamotene & 1496 & 1480 & - & 0.1 & - & - & - & - \\
\hline 56 & $\alpha$-Selinene & 1498 & 1498 & - & - & - & 0.5 & - & - \\
\hline 57 & Germacrene D & 1498 & 1484 & 0.3 & 0.1 & 2.7 & - & - & - \\
\hline 58 & Bicyclogermacrene & 1513 & 1500 & 0.7 & 0.6 & 1 & - & 0.2 & 0.2 \\
\hline 59 & $\beta$-Bisabolene & 1517 & 1505 & - & 0.2 & - & - & - & - \\
\hline 60 & Eugenyl acetate & 1533 & 1521 & 9.5 & 9.3 & 0.1 & - & - & - \\
\hline 61 & $\delta$-Cadinene & 1537 & 1522 & 0.2 & 0.3 & 0.2 & - & - & 0.1 \\
\hline 62 & (E)- $\alpha$-Bisabolene & 1551 & 1544 & - & 0.3 & - & - & - & - \\
\hline 63 & (E)-Nerolidol & 1570 & 1561 & - & - & 0.3 & 0.9 & - & - \\
\hline 64 & Germacrene B & 1577 & 1559 & - & - & 0.2 & - & - & - \\
\hline 65 & Spathulenol & 1598 & 1577 & - & 0.6 & - & 0.4 & 1.1 & 0.8 \\
\hline
\end{tabular}


Table 2. Cont.

\begin{tabular}{|c|c|c|c|c|c|c|c|c|c|}
\hline $\mathrm{N}^{\mathbf{o}}$ & Compounds & $\mathbf{R I}^{\mathrm{a}}$ & $\mathbf{R I}^{\mathrm{b}}$ & C. ov. ${ }^{\mathrm{c}}$ & C. ov. ${ }^{\mathrm{c}}$ & C.to. ${ }^{\mathrm{d}}$ & C. $d a .^{\mathrm{e}}$ & C. $\operatorname{lo}^{\mathrm{f}}$ & C.po..$^{\mathrm{g}}$ \\
\hline & & & & Leaf & Stem & Leaf & Leaf & Leaf & Leaf \\
\hline 66 & Caryophyllene oxide & 1605 & 1582 & - & 0.5 & 0.2 & 1.1 & 0.5 & 0.9 \\
\hline 67 & Intermedeol isomer & 1616 & - & - & - & - & 5.8 & - & - \\
\hline 68 & Selin-11-en-4-one & 1626 & 1626 & - & - & - & 1.5 & - & - \\
\hline 69 & Selina-3,11-dien- $6 \alpha$-ol & 1644 & 1642 & - & - & - & 0.6 & - & - \\
\hline 70 & $\alpha$-Cadinol & 1652 & 1652 & - & - & - & 0.5 & - & - \\
\hline 71 & Selin-11-en- $4 \alpha$-ol & 1660 & 1658 & - & - & - & 7.3 & - & - \\
\hline 72 & Germacra-4(15),5,10(14)-trien-1 $\alpha$-ol & 1685 & 1685 & - & - & - & 1 & - & - \\
\hline 73 & Aromadendrane-4,10-diol & 1717 & - & - & - & - & 0.5 & - & - \\
\hline 74 & Oplopanone & 1735 & 1739 & - & - & - & 0.3 & - & - \\
\hline 75 & $\alpha$-Cyperone & 1747 & - & - & - & - & 4 & - & - \\
\hline \multirow[t]{8}{*}{76} & Cyclocolorenone & 1763 & 1759 & - & - & - & 0.1 & - & - \\
\hline & Monoterpene hydrocarbons & & & 7.3 & 4.7 & 46.6 & 2.5 & 7.4 & 17.9 \\
\hline & Oxygenated monoterpenoids & & & 6.6 & 9.4 & 44.7 & 46.5 & 90.1 & 72.3 \\
\hline & Sesquiterpene hydrocarbons & & & 4.1 & 3.1 & 7.5 & 21.1 & 0.2 & 1.9 \\
\hline & Oxygenated sesquiterpenoids & & & 0 & 1.1 & 0.5 & 24 & 1.6 & 1.7 \\
\hline & Phenylpropanoids & & & 80 & 81 & 0.3 & 0.1 & 0 & 0.9 \\
\hline & Others & & & 0 & 0 & 0.2 & 0 & 0 & 0.2 \\
\hline & Total identified & & & 98 & 99.3 & 99.8 & 94.2 & 99.3 & 94.9 \\
\hline
\end{tabular}

\footnotetext{
${ }^{a} \mathrm{RI}=$ Retention Index determined on an HP-5ms column. ${ }^{\mathrm{b}} \mathrm{RI}$ from the databases. ${ }^{\mathrm{c}} \mathrm{C} . \mathrm{ov} .=$ Cinnamomum ovatum

${ }^{\mathrm{d}} \mathrm{C}$. to. = Cinnamomum tonkinense. ${ }^{\mathrm{e}} \mathrm{C}$. da. = Cinnamomum damhaensis. ${ }^{\mathrm{f}} \mathrm{C}$. lo. = Cinnamomum longipetiolatum.

g C. po. = Cinnamomum polyadelphum.
}

\subsection{Antimicrobial Screening}

The Cinnamomum essential oils were screened for antimicrobial activity against Gram-positive (Enterococcus faecalis, Staphylococcus aureus, Bacillus cereus) and Gram-negative (Escherichia coli, Pseudomonas aeruginosa, Salmonella enterica) bacteria and a yeast (Candida albicans). Minimum inhibitory concentrations and $\mathrm{IC}_{50}$ values were determined using the microbroth dilution assay (Table 3).

Table 3. Antimicrobial activities of Cinnamomum essential oils from north central Vietnam.

\begin{tabular}{|c|c|c|c|c|c|c|c|}
\hline Sample & $\begin{array}{l}\text { Enterococcus } \\
\text { faecalis }\end{array}$ & $\begin{array}{c}\text { Gram-Positive } \\
\text { Staphylococcus } \\
\text { aureus }\end{array}$ & $\begin{array}{c}\text { Bacillus } \\
\text { cereus }\end{array}$ & $\begin{array}{l}\text { Escherichia } \\
\text { coli }\end{array}$ & $\begin{array}{c}\text { Gram-Negative } \\
\text { Pseudomonas } \\
\text { aeruginosa }\end{array}$ & $\begin{array}{c}\text { Salmonella } \\
\text { enterica }\end{array}$ & $\begin{array}{c}\text { Yeast } \\
\text { Candida } \\
\text { albicans }\end{array}$ \\
\hline & \multicolumn{7}{|c|}{$\operatorname{MIC}\left(\mu \mathrm{g} / \mathrm{mL}^{\mathrm{a}}\right)$} \\
\hline C. ovatum leaf & 64 & 64 & 128 & 64 & 128 & 64 & 64 \\
\hline C. ovatum stem & 64 & 64 & 64 & 64 & 16 & 64 & 32 \\
\hline C. tonkinense & 32 & 128 & 128 & - & - & - & 32 \\
\hline C. damhaensis & - & - & - & - & - & - & - \\
\hline C. longipetiolatum & 64 & 128 & 128 & 256 & 256 & 128 & 256 \\
\hline C. polyadelphum & 32 & 64 & 64 & - & - & 128 & 256 \\
\hline Streptomycin & 32 & 128 & 64 & 32 & 128 & 64 & - \\
\hline Nistatin & - & - & - & - & - & - & 8 \\
\hline \multirow[t]{2}{*}{ Cyclohexamide } & - & - & - & - & - & - & 32 \\
\hline & \multicolumn{7}{|c|}{$\mathrm{IC}_{50}\left(\mu \mathrm{g} / \mathrm{mL}^{\mathrm{a}}\right)$} \\
\hline C. ovatum leaf & 32.33 & 32.33 & 65.45 & 32.56 & 65.44 & 33.22 & 33.22 \\
\hline C. ovatum stem & 32.44 & 32.78 & 33.56 & 32.56 & 8.77 & 31.22 & 15.67 \\
\hline C. tonkinense & 5.67 & 37.78 & 56.67 & - & - & - & 15.67 \\
\hline C. damhaensis & - & - & - & - & - & - & - \\
\hline C. longipetiolatum & 17.88 & 36.78 & 56.79 & 100.34 & 105.67 & 56.78 & 112.45 \\
\hline C. polyadelphum & 10.67 & 24.78 & 30.24 & - & - & 57.45 & 123.45 \\
\hline
\end{tabular}

${ }^{a}$ Micrograms of essential oil per milliliter of test solution.

\subsection{Larvicidal Screening}

The Cinnamomum essential oils were screened for mosquito larvicidal activity against Aedes aegypti, Aedes albopictus and Culex quinquefasciatus. The $24 \mathrm{~h}$ and $48 \mathrm{~h} \mathrm{LC} 50$ and $\mathrm{LC}_{90}$ values are summarized in Tables 4 and 5. 
Table 4. Twenty-four-hour mosquito larvicidal activities $(\mu \mathrm{g} / \mathrm{mL})$ of Cinnamomum essential oils from north central Vietnam.

\begin{tabular}{|c|c|c|c|c|}
\hline Sample & LC $_{50}(95 \%$ Confidence Limits $)$ & LC $_{90}(95 \%$ Confidence Limits $)$ & $x^{2}$ & $p$ \\
\hline \multicolumn{5}{|c|}{ Aedes aegypti } \\
\hline C. ovatum leaf EO & $24.12(20.92-27.45)$ & $50.61(45.02-58.65)$ & 48.86 & 0.000 \\
\hline C. ovatum stem EO & $52.51(48.77-57.69)$ & $71.23(64.50-82.64)$ & 0.4722 & 0.790 \\
\hline C. tonkinensis leaf EO & $17.44(15.53-19.58)$ & $31.40(27.93-36.64)$ & 0.1354 & 0.987 \\
\hline C. damhaensis leaf EO & $21.43(18.66-24.15)$ & $38.98(34.75-45.58)$ & 0.5494 & 0.760 \\
\hline C. longepetiolatum leaf EO & $64.20(55.67-73.61)$ & $127.9(111.0-156.5)$ & 8.805 & 0.003 \\
\hline C. polyadelphum leaf EO & $23.41(21.37-25.78)$ & $36.69(33.27-41.52)$ & 8.277 & 0.041 \\
\hline \multicolumn{5}{|c|}{ Aedes albopictus } \\
\hline C. ovatum leaf EO & n.t. & n.t. & - & - \\
\hline C. ovatum stem EO & $61.45(55.66-68.20)$ & $103.3(93.3-117.1)$ & 34.38 & 0.000 \\
\hline C. tonkinensis leaf EO & $42.89(39.73-46.59)$ & $61.65(56.52-69.09)$ & 2.595 & 0.273 \\
\hline C. damhaensis leaf EO & $43.91(41.25-46.46)$ & $56.16(52.95-60.79)$ & 0.04480 & 0.978 \\
\hline C. longepetiolatum leaf EO & n.t. & n.t. & - & - \\
\hline C. polyadelphum leaf EO & $20.66(18.02-23.28)$ & $37.21(33.04-43.97)$ & 2.577 & 0.276 \\
\hline \multicolumn{5}{|c|}{ Culex quinquefasciatus } \\
\hline C. ovatum leaf EO & $34.19(31.18-37.65)$ & $56.01(50.85-63.12)$ & 10.73 & 0.013 \\
\hline C. ovatum stem EO & $28.79(22.07-34.79)$ & $78.3(67.72-94.57$ & 8.295 & 0.016 \\
\hline C. tonkinensis leaf EO & $14.05(12.28-15.75)$ & $25.70(23.06-29.59)$ & 16.31 & 0.001 \\
\hline C. damhaensis leaf EO & $46.74(41.58-52.63)$ & 86.80 (77.37-100.39) & 13.53 & 0.001 \\
\hline C. longepetiolatum leaf EO & $126.8(108.3-151.4)$ & $293.9(248.2-368.9)$ & 21.47 & 0.000 \\
\hline C. polyadelphum leaf EO & $18.33(13.26-22.87)$ & $58.95(50.62-72.05)$ & 5.639 & 0.131 \\
\hline
\end{tabular}

Table 5. Forty-eight-hour mosquito larvicidal activities $(\mu \mathrm{g} / \mathrm{mL})$ of Cinnamomum essential oils from north central Vietnam.

\begin{tabular}{|c|c|c|c|c|}
\hline Sample & LC $_{50}(95 \%$ Confidence Limits $)$ & LC $_{90}(95 \%$ Confidence Limits $)$ & $x^{2}$ & $p$ \\
\hline \multicolumn{5}{|c|}{ Aedes aegypti } \\
\hline C. ovatum leaf EO & $13.76(11.42-15.95)$ & $30.17(26.76-35.17)$ & 46.16 & 0.000 \\
\hline C. ovatum stem EO & $46.74(43.17-51.21)$ & $67.53(61.30-77.07)$ & 2.744 & 0.254 \\
\hline C. tonkinensis leaf EO & $15.83(13.76-17.99)$ & $31.17(27.53-36.80)$ & 2.196 & 0.533 \\
\hline C. damhaensis leaf EO & $17.36(13.67-20.36)$ & $37.53(32.94-45.22)$ & 5.494 & 0.064 \\
\hline C. longepetiolatum leaf EO & $39.50(29.92-47.24)$ & $95.24(83.27-114.77)$ & 2.513 & 0.113 \\
\hline C. polyadelphum leaf EO & $17.30(15.44-19.41)$ & $30.80(27.43-35.89)$ & 3.650 & 0.302 \\
\hline \multicolumn{5}{|c|}{ Aedes albopictus } \\
\hline C. ovatum leaf EO & n.t. & n.t. & - & - \\
\hline C. ovatum stem EO & $50.18(45.07-56.12)$ & $87.98(78.88-100.81)$ & 35.66 & 0.000 \\
\hline C. tonkinensis leaf EO & $42.74(39.48-46.59)$ & $62.40(57.00-70.25)$ & 4.098 & 0.129 \\
\hline C. damhaensis leaf EO & $39.85(37.05-42.91)$ & $56.02(51.91-61.70)$ & 0.06006 & 0.970 \\
\hline C. longepetiolatum leaf EO & n.t. & n.t. & - & - \\
\hline C. polyadelphum leaf EO & $20.79(17.84-23.61)$ & $39.45(34.97-46.59)$ & 6.980 & 0.031 \\
\hline \multicolumn{5}{|c|}{ Culex quinquefasciatus } \\
\hline C. ovatum leaf EO & $30.48(27.00-34.48)$ & $59.19(52.54-68.81)$ & 1.181 & 0.757 \\
\hline C. ovatum stem EO & $20.54(11.92-27.10)$ & $72.40(62.01-89.54)$ & 5.799 & 0.055 \\
\hline C. tonkinensis leaf EO & $8.721(6.874-10.253)$ & $18.81(16.70-22.01)$ & 26.83 & 0.000 \\
\hline C. damhaensis leaf EO & $18.63(9.90-25.06)$ & $67.93(58.16-84.15)$ & 6.243 & 0.001 \\
\hline C. longepetiolatum leaf EO & 76.88 (52.08-101.93) & $314.5(249.3-447.0)$ & 47.36 & 0.000 \\
\hline C. polyadelphum leaf EO & 11.03 (4.50-15.93) & $52.40(44.35-65.76)$ & 10.30 & 0.016 \\
\hline
\end{tabular}

\section{Discussion}

\subsection{Cinnamomum ovatum}

The leaf and stem bark essential oils of C. ovatum demonstrated broad antimicrobial activity against the organisms tested with MIC values ranging from 16 to $128 \mu \mathrm{g} / \mathrm{mL}$ (Table 3). The major components of the leaf and stem essential oils were eugenol (70.5\% and $71.2 \%$, respectively), eugenyl acetate $(9.5 \%$ and $9.3 \%$, respectively) and linalool (5.9\% and $8.3 \%$, respectively) (Table 2$)$. The high concentration of eugenol in these two essential oils is likely responsible for the observed antimicrobial effects. Eugenol has shown broad spectrum antibacterial [35,36] and antifungal [37-39] activities. Likewise, the mosquito larvicidal activity of C. ovatum leaf essential oil is likely due to eugenol; that compound has shown larvicidal activity against Ae. aegypti [40], Ae. albopictus [41] and Cx. quinquefasciatus [42]. 
Cinnamomum cambodianum leaf [34] and stem bark [33] essential oils from Vietnam have also shown high concentrations of linalool ( $27.0 \%$ and $33.1 \%$, respectively).

\subsection{Cinnamomum tonkinense}

Cinnamomum tonkinense leaf essential oil showed excellent antimicrobial activity against $E$. faecalis and C. albicans with MIC of $32 \mu \mathrm{g} / \mathrm{mL}$ and good activity against B. cereus and S. aureus (Table 3). The essential oil is rich in monoterpenes, $\alpha$-pinene $(4.0 \%)$, sabinene $(3.4 \%), \alpha$-phellandrene $(4.8 \%)$, $\beta$-phellandrene (23.1\%), 1,8-cineole (9.8\%), linalool (32.2\%) (Table 2). Both $\alpha$-pinene and linalool have shown antibacterial activity against $E$. faecalis [35] and S. aureus [43]; $\alpha$-pinene and 1,8-cineole have shown antifungal activity against $C$. albicans [43]. Sabinene, on the other hand, has shown little [44] or no [45] antimicrobial activity. Likewise, $\alpha$-phellandrene has shown no activity against C. albicans [46]. The leaf essential oils of $C$. cordatum and C. scortechini from Pahang, Malaysia, both rich in $\beta$-phellandrene $(9.0 \%$ and $17.3 \%$, respectively) and linalool (17.3\% and $16.4 \%$, respectively), have shown antifungal activities against several fungal strains [47].

The leaf essential oil of $C$. tonkinense is one of the most larvicidal in this study (Tables 4 and 5). The major components in the essential oil likely account for the observed larvicidal activity. $\alpha$-Pinene, has been shown to be larvicidal against Ae. aegypti, Ae. albopictus and $C x$. quinquefasciatus [48]; sabinene and linalool have both demonstrated larvicidal against $A$ e. aegypti and $C x$. quinquefasciatus [49]; and $\alpha$-phellandrene has shown activity against Ae. aegypti and Ae. albopictus [50] as well as Culex pipiens molestus [51]. The leaf essential oil of $C$. scortechinii, rich in $\beta$-phellandrene (17.3\%) and linalool (16.4\%), had shown excellent larvicidal activity against Ae. aegypti and Ae. albopictus $\left(\mathrm{LC}_{50}=21.5\right.$ and $16.7 \mu \mathrm{g} / \mathrm{mL}$, respectively) [52].

\subsection{Cinnamomum damhaensis}

The major components of $C$. damhaensis leaf essential oil were linalool $(44.8 \%)$ and $\beta$-selinene (19.1\%) (Table 2). The essential oil also showed pronounced larvicidal activity against Ae. aegypti and $C x$. quinquefasciatus with $48 \mathrm{~h} \mathrm{LC}_{50}$ values of 17.4 and $18.6 \mu \mathrm{g} / \mathrm{mL}$, respectively (Table 5), which can be attributed to the high concentration of linalool (see above). Note that Piper gaudichaudianum and Piper humaytanum leaf essential oils, rich in $\beta$-selinene (10.5\% and $15.8 \%$, respectively), but devoid of linalool, showed only marginal larvicidal activity against Ae. aegypti [53].

\subsection{Cinnamomum longipetiolatum}

The leaf essential oil of $C$. longipetiolatum was dominated by linalool $(75.7 \%$, Table 2$)$, which likely accounts for the observed antimicrobial (Table 3) activity; linalool has shown broad antibacterial and antifungal activity $[35,54]$. Although linalool has shown larvicidal activity against Ae. aegypti $\left(\mathrm{LC}_{50}=38.6 \mu \mathrm{g} / \mathrm{mL}\right)$ and $C x$. quinquefasciatus $\left(\mathrm{LC}_{50}=42.3 \mu \mathrm{g} / \mathrm{mL}\right)$ [49], the larvicidal activity of C. longipetiolatum leaf oil was less $\left(24 \mathrm{~h} \mathrm{LC}_{50}=64.2\right.$ and $126.8 \mu \mathrm{g} / \mathrm{mL}$ against Ae. aegypti and Cx. quinquefasciatus, respectively, Table 4).

\subsection{Cinnamomum polyadelphum}

The leaf essential oil of $C$. polyadelphum showed good activity against the Gram-positive organisms tested with MIC values of 32, 64 and $64 \mu \mathrm{g} / \mathrm{mL}$ on E. faecalis, S. aureus and B. cereus, respectively (Table 3). The essential oil also showed notable larvicidal activity against all three mosquito species with 48-h $\mathrm{LC}_{50}$ values of $17.3,20.8$ and $11.0 \mu \mathrm{g} / \mathrm{mL}$ against Ae. aegypti, Ae. albopictus and Cx. quinquefasciatus, respectively (Table 5). The major components in C. polyadelphum leaf essential oil were camphor $(32.2 \%)$, neral $(11.7 \%)$ and geranial $(16.6 \%)$ (Table 2$)$. The antimicrobial properties of camphor are relatively marginal $[55,56]$. Citral (mixture of neral and geranial), on the other hand, has shown greater antimicrobial activity on Gram-positive bacteria [57-59] and fungi [60,61]. Likewise, citral has exhibited mosquito larvicidal activity against Ae. albopictus [62] but camphor is inactive against larvae of Ae. aegypti, Ae. albopictus [52,62] or Cx. pipiens [63]. 


\section{Materials and Methods}

\subsection{Plant Material}

Leaves or stem bark of the Cinnamomum species were collected from locations in north central Vietnam (see Table 1). Plants were identified by Do N. Dai and voucher specimens (Table 1) have been deposited in the plant specimen room, Faculty Agriculture, Forestry and Fishery, Nghe An, College of Economics. The fresh plant materials $(2.0 \mathrm{~kg}$ each) were shredded and hydrodistilled using a Clevenger apparatus for $4 \mathrm{~h}$ to give the essential oils. The essential oil yields are summarized in Table 1.

\subsection{Gas Chromatographic Analysis}

Gas chromatography (GC) analysis was performed on an Agilent Technologies (Santa Clara, CA, USA) HP 7890A Plus Gas chromatograph equipped with a flame ionization detector (FID) and fitted with HP-5ms column (30 $\mathrm{m} \times 0.25 \mathrm{~mm}$, film thickness $0.25 \mu \mathrm{m}$, Agilent Technologies). The analytical conditions were-carrier gas $\mathrm{H}_{2}(1 \mathrm{~mL} / \mathrm{min})$, injector temperature (PTV) $250{ }^{\circ} \mathrm{C}$, detector temperature $260{ }^{\circ} \mathrm{C}$, column temperature programmed from $60{ }^{\circ} \mathrm{C}(2 \mathrm{~min}$ hold $)$ to $220^{\circ} \mathrm{C}(10 \mathrm{~min}$ hold $)$ at $4{ }^{\circ} \mathrm{C} / \mathrm{min}$. Samples were injected by splitting and the split ratio was 10:1. The volume injected was $1.0 \mu \mathrm{L}$. Inlet pressure was $6.1 \mathrm{kPa}$.

An Agilent Technologies (Santa Clara, California, USA) HP 7890A Plus Chromatograph fitted with a fused silica capillary HP-5ms (30 m $\times 0.25 \mathrm{~mm}$, film thickness $0.25 \mu \mathrm{m})$ and interfaced with a mass spectrometer (HP 5973 MSD) was used for the GC-MS analysis, under the same conditions as those used for GC-FID analysis. The conditions were the same as described above with He (1 mL/min) as carrier gas. The MS conditions were as follows-ionization voltage $70 \mathrm{eV}$; emission current $40 \mathrm{~mA}$; acquisitions scan mass range of 35-350 amu at a sampling rate of $1.0 \mathrm{scan} / \mathrm{s}$.

The identification of constituents was performed on the basis of retention indices (RI) determined with reference to a homologous series of $n$-alkanes, under identical experimental conditions, co-injection with standards (Sigma-Aldrich, St. Louis, MO, USA) or known essential oil constituents, MS library search (NIST 08 and Wiley $9^{\text {th }}$ Version) and by comparing with MS literature data [64]. The relative amounts of individual components were calculated based on the GC peak area (FID response) without using correction factors.

\subsection{Antimicrobial Screening}

The antimicrobial activity of the essential oils was evaluated using three strains of Gram-positive test bacteria, Enterococcus faecalis (ATCC299212), Staphylococcus aureus (ATCC25923), Bacillus cereus (ATCC14579), three strains of Gram-negative test bacteria, Escherichia coli (ATCC 25922), Pseudomonas aeruginosa (ATCC27853), Salmonella enterica (ATCC13076) and one strain of yeast, Candida albicans (ATCC 10231).

Minimum inhibitory concentration (MIC) and median inhibitory concentration $\left(\mathrm{IC}_{50}\right)$ values were measured by the microdilution broth susceptibility assay [65]. Stock solutions of the oil were prepared in dimethylsulfoxide. Dilution series were prepared from 16,384 to $2 \mu \mathrm{g} / \mathrm{mL}\left(2^{14}, 2^{13}, 2^{12}, 2^{11}, 2^{10}, 2^{9}, 2^{7}\right.$, $2^{5}, 2^{3}$ and $2^{1} \mu \mathrm{g} / \mathrm{mL}$ ) in sterile distilled water in micro-test tubes from where they were transferred to 96-well microtiter plates. Bacteria grown in double-strength Mueller-Hinton broth or double-strength tryptic soy broth and fungi grown in double-strength Sabouraud dextrose broth were standardized to $5 \times 10^{5}$ and $1 \times 10^{3} \mathrm{CFU} / \mathrm{mL}$, respectively. The last row, containing only the serial dilutions of sample without microorganisms, was used as a positive (no growth) control. Sterile distilled water and medium served as a negative (no antimicrobial agent) control. Streptomycin was used as the antibacterial standard, nystatin and cycloheximide were used as antifungal standards. After incubation at $37^{\circ} \mathrm{C}$ for $24 \mathrm{~h}$, the MIC values were determined to be the well with the lowest concentration of agents completely inhibiting the growth of microorganisms. The $\mathrm{IC}_{50}$ values were determined by the percentage of microorganisms that inhibited growth based on the turbidity measurement data 
of EPOCH2C spectrophotometer (BioTeK Instruments, Inc Highland Park Winooski, VT, USA) and Rawdata computer software (Brussels, Belgium) according to the following equations:

$$
\begin{gathered}
\%_{\text {inhibition }}=\frac{O D_{\text {control }(-)}-O D_{\text {test agent }}}{O D_{\text {control }(-)}-O D_{\text {control }(+)}} \\
I C_{50}=H i g h_{\text {conc }}-\frac{\left(H i g h_{\text {inh } \%}-50 \%\right) \times\left(H i g h_{\text {conc }}-L o w w_{\text {conc }}\right)}{\left(H i g h_{i n h} \%-L o w_{\text {inh } \%}\right)}
\end{gathered}
$$

where OD is the optical density, control(-) are the cells with medium but without antimicrobial agent, test agent corresponds to a known concentration of antimicrobial agent, control(+) is the culture medium without cells, $\mathrm{High}_{\text {conc }} / \mathrm{Low}_{\text {conc }}$ is the concentration of test agent at high concentration/low concentration and $\mathrm{High}_{\mathrm{inh}} / \mathrm{Low}_{\mathrm{inh}} \%$ is the $\%$ inhibition at high concentration/\% inhibition at low concentration). Each of the antimicrobial screens were carried out in triplicate.

\subsection{Larvicidal Screening}

Eggs of Aedes aegypti were purchased from Institute of Biotechnology, Vietnam Academy of Science and Technology and maintained at the Laboratory of Department of Pharmacy of Duy Tan University, Da Nang, Vietnam. Adults of Culex quinquefasciatus and Aedes albopictus collected in Hoa Khanh Nam ward, Lien Chieu district, Da Nang city $\left(16^{\circ} 03^{\prime} 14.9^{\prime \prime} \mathrm{N}, 108^{\circ} 09^{\prime} 31.2^{\prime \prime} \mathrm{E}\right)$ and were identified by National institute of Malariology, Parasitology and Entomology, Ho Chi Minh City. Adult mosquitoes were maintained in entomological cages $(40 \times 40 \times 40 \mathrm{~cm})$ and fed a $10 \%$ sucrose solution and were allowed to blood feed on 1-week-old chicks and mice, respectively. Egg hatchings were induced with tap water. Larvae were reared in plastic trays $(24 \times 35 \times 5 \mathrm{~cm})$. The larvae were fed on Koi fish food. All developmental stages were maintained at $25 \pm 2{ }^{\circ} \mathrm{C}, 65-75 \%$ relative humidity and a $12: 12 \mathrm{~h}$ light:dark cycle at the Laboratory of the Faculty of Environmental and Chemical Engineering of Duy Tan University, Da Nang, Vietnam.

Larvicidal activities of the Cinnamomum essential oils were evaluated according to the protocol Liu and co-workers [66] with slight modifications. For the assay, $150 \mathrm{~mL}$ of water that contained 20 larvae (fourth instar) was placed in 250-mL beakers and aliquots of the Cinnamomum essential oils dissolved in $\mathrm{EtOH}$ ( $1 \%$ stock solution) were then added. With each experiment, a set of controls using EtOH only (negative control) and permethrin (positive control) were also run for comparison. Mortality was recorded after $24 \mathrm{~h}$ and again after $48 \mathrm{~h}$ of exposure during which no nutritional supplement was added. The experiments were carried out at $25 \pm 2{ }^{\circ} \mathrm{C}$. Each test was conducted with four replicates with five concentrations $(100,50,25,12.5$ and $6 \mu \mathrm{g} / \mathrm{mL})$. The data obtained were subjected to log-probit analysis [67] to obtain $\mathrm{LC}_{50}$ values, $\mathrm{LC}_{90}$ values and $95 \%$ confidence limits using Minitab ${ }^{\circledR} 19$ (Minitab, LLC, State College, PA, USA).

\section{Conclusions}

The essential oils of five species of Cinnamomum were collected from north central Vietnam and screened for antimicrobial and mosquito larvicidal activities. According to Duarte and co-workers [68], essential oils with MIC values between 50 and $500 \mu \mathrm{g} / \mathrm{mL}$ can be considered to have strong antimicrobial activity. Similarly, Dias and Moraes have concluded that essential oils with $\mathrm{LC}_{50}<100 \mu \mathrm{g} / \mathrm{mL}$ are considered to be active [69]. Therefore, all of the Cinnamomum essential oils in this study can be considered to be active and show promise as antimicrobial agents and as alternative insecticidal agents against mosquito larvae.

Author Contributions: Conceptualization, D.N.D. and W.N.S.; methodology, D.N.D., L.T.H., N.H.H., W.N.S.; validation, D.N.D. and W.N.S.; formal analysis, L.T.H., W.N.S.; investigation, N.T.C., L.T.H., N.T.Y., D.T.M.C.; resources, D.N.D.; data curation, W.N.S.; writing—original draft preparation, W.N.S., D.N.D.; writing-review and editing, D.N.D., L.T.H., W.N.S.; supervision, D.N.D.; project administration, D.N.D.; funding acquisition, D.N.D. All authors have read and agreed to the published version of the manuscript. 
Funding: This research was funded by Vietnam National Foundation for Science and Technology Development (NAFOSTED) under grant number: 106.03-2018.02.

Acknowledgments: W.N.S. participated in this work as part of the activities of the Aromatic Plant Research Center (APRC, https://aromaticplant.org/).

Conflicts of Interest: The authors declare no conflict of interest.

\section{References}

1. Mabberley, D.J. Mabberley's Plant-Book, 3rd ed.; Cambridge University Press: Cambridge, UK, 2008.

2. Ho, P.-H. An Illustrated Flora of Vietnam, Vol. 3; Youth Publishing House: Ho Chi Minh City, Vietnam, 2000.

3. Dao, N.K. Flora of Vietnam, Lauraceae Juss, Vol. 17; Publishing House for Science \& Technology: Hanoi, Vietnam, 2017.

4. Chi, V.V. Dictionary of Vietnamese Medicinal Plants, Vol. I-II; Medical Publishing House: Hanoi, Vietnam, 2012.

5. Moi, L.D.; Cu, L.D.; Hoi, T.M.; Thuy, N.T.; Thao, N.T.; Thai, T.H.; Ban, N.K. The Essential Oil Resources in Vietnam, Vol. 1; Agriculture Publishing House: Hanoi, Vietnam, 2001.

6. Zaman, S.B.; Hussain, M.A.; Nye, R.; Mehta, V.; Mamun, K.T.; Hossain, N. A review on antibiotic resistance: Alarm bells are ringing. Cureus 2017, 9, e1403. [CrossRef] [PubMed]

7. Banin, E.; Hughes, D.; Kuipers, O.P. Bacterial pathogens, antibiotics and antibiotic resistance. FEMS Microbiol. Rev. 2017, 41, 450-452. [CrossRef] [PubMed]

8. Navon-Venezia, S.; Kondratyeva, K.; Carattoli, A. Klebsiella pneumoniae: A major worldwide source and shuttle for antibiotic resistance. FEMS Microbiol. Rev. 2017, 41, 252-275. [CrossRef] [PubMed]

9. Pierce, G.E. Pseudomonas aeruginosa, Candida albicans and device-related nosocomial infections: Implications, trends and potential approaches for control. J. Ind. Microbiol. Biotechnol. 2005, 32, 309-318. [CrossRef] [PubMed]

10. Sakoulas, G.; Moellering, R.C., Jr. Increasing antibiotic resistance among methicillin-resistant Staphylococcus aureus strains. Clin. Infect. Dis. 2008, 46, S360-S367. [CrossRef] [PubMed]

11. Sweileh, W.M.; Sawalha, A.F.; Al-Jabi, S.; Zyoud, S.H. Bibliometric analysis of literature on antifungal triazole resistance: 1980-2015. Germs 2017, 7, 19-27. [CrossRef]

12. Rajkowska, K.; Kunicka-Styczyńska, A.; Maroszyńska, M. Selected essential oils as antifungal agents against antibiotic-resistant Candida spp.: In vitro study on clinical and food-borne isolates. Microb. Drug Resist. 2017, 23, 18-24. [CrossRef]

13. Orchard, A.; van Vuuren, S. Commercial essential oils as potential antimicrobials to treat skin diseases. Evid. Based Complement. Altern. Med. 2017, 2017, 4517971. [CrossRef]

14. Orchard, A.; Viljoen, A.; van Vuuren, S. Wound pathogens: Investigating antimicrobial activity of commercial essential oil combinations against reference strains. Chem. Biodivers. 2018, 15, e1800405. [CrossRef]

15. Serra, E.; Hidalgo-Bastida, L.A.; Verran, J.; Williams, D.; Malic, S. Antifungal activity of commercial essential oils and biocides against Candida albicans. Pathogens 2018, 7, 15. [CrossRef]

16. Powers, C.N.; Osier, J.L.; McFeeters, R.L.; Brazell, C.B.; Olsen, E.L.; Moriarity, D.M.; Satyal, P.; Setzer, W.N. Antifungal and cytotoxic activities of sixty commercially-available essential oils. Molecules 2018, 23, 1549. [CrossRef] [PubMed]

17. Wińska, K.; Mączka, W.; Łyczko, J.; Grabarczyk, M.; Czubaszek, A.; Szumny, A. Essential oils as antimicrobial agents-Myth or real alternative? Molecules 2019, 24, 2130. [CrossRef] [PubMed]

18. D'agostino, M.; Tesse, N.; Frippiat, J.P.; Machouart, M.; Debourgogne, A. Essential oils and their natural active compounds presenting antifungal properties. Molecules 2019, 24, 3713. [CrossRef] [PubMed]

19. Kozics, K.; Bučková, M.; Puškárová, A.; Kalászová, V.; Cabicarová, T.; Pangallo, D. The Effect of ten essential oils on several cutaneous drug-resistant microorganisms and their cyto/genotoxic and antioxidant properties. Molecules 2019, 24, 4570. [CrossRef] [PubMed]

20. Leta, S.; Beyene, T.J.; De Clercq, E.M.; Amenu, K.; Kraemer, M.U.G.; Revie, C.W. Global risk mapping for major diseases transmitted by Aedes aegypti and Aedes albopictus. Int. J. Infect. Dis. 2018, 67, 25-35. [CrossRef] [PubMed]

21. Samy, A.M.; Elaagip, A.H.; Kenawy, M.A.; Ayres, C.F.J.; Peterson, A.T.; Soliman, D.E. Climate change influences on the global potential distribution of the mosquito Culex quinquefasciatus, vector of West Nile virus and lymphatic filariasis. PLoS ONE 2016, 11, e0163863. [CrossRef] 
22. Song, S.; Li, Y.; Fu, S.; Liu, H.; Li, X.; Gao, X.; Xu, Z.; Liu, G.; Wang, D.; Tian, Z.; et al. Could Zika virus emerge in Mainland China? Virus isolation from nature in Culex quinquefasciatus, 2016. Emerg. Microbes Infect. 2017, 6, e93. [CrossRef]

23. Bhattacharya, S.; Basu, P. The southern house mosquito, Culex quinquefasciatus: Profile of a smart vector. J. Entomol. Zool. Stud. 2016, 4, 73-81.

24. Cook, C.L.; Huang, Y.-J.S.; Lyons, A.C.; Alto, B.W.; Unlu, I.; Higgs, S.; Vanlandingham, D.L. North American Culex pipiens and Culex quinquefasciatus are competent vectors for Usutu virus. PLoS Negl. Trop. Dis. 2018, 12, e0006732. [CrossRef]

25. Naqqash, M.N.; Gökçe, A.; Bakhsh, A.; Salim, M. Insecticide resistance and its molecular basis in urban insect pests. Parasitol. Res. 2016, 115, 1363-1373. [CrossRef]

26. Grisales, N.; Poupardin, R.; Gomez, S.; Fonseca-Gonzalez, I.; Ranson, H.; Lenhart, A. Temephos resistance in Aedes aegypti in Colombia compromises dengue vector control. PLoS Negl. Trop. Dis. 2013, 7, e2438. [CrossRef]

27. Elia-Amira, N.M.R.; Chen, C.D.; Lau, K.W.; Lee, H.L.; Low, V.L.; Norma-Rashid, Y.; Sofian-Azirun, M. Organophosphate and organochlorine resistance in larval stage of Aedes albopictus (Diptera: Culicidae) in Sabah, Malaysia. J. Econ. Entomol. 2018, 111, 2488-2492. [CrossRef]

28. Delisi, N.; Ottea, J.; Healy, K. Susceptibility of Culex quinquefasciatus (Diptera: Culicidae) in southern Louisiana to larval insecticides. J. Econ. Entomol. 2017, 110, 2562-2567. [CrossRef]

29. Pitasawat, B.; Champakaew, D.; Choochote, W.; Jitpakdi, A.; Chaithong, U.; Kanjanapothi, D.; Rattanachanpichai, E.; Tippawangkosol, P.; Riyong, D.; Tuetun, B.; et al. Aromatic plant-derived essential oil: An alternative larvicide for mosquito control. Fitoterapia 2007, 78, 205-210. [CrossRef]

30. Silva, W.J.; Dória, G.A.A.; Maia, R.T.; Nunes, R.S.; Carvalho, G.A.; Blank, A.F.; Alves, P.B.; Marçal, R.M.; Cavalcanti, S.C.H. Effects of essential oils on Aedes aegypti larvae: Alternatives to environmentally safe insecticides. Bioresour. Technol. 2008, 99, 3251-3255. [CrossRef]

31. Pavela, R. Essential oils for the development of eco-friendly mosquito larvicides: A review. Ind. Crop. Prod. 2015, 76, 174-187. [CrossRef]

32. Son, L.C.; Dai, D.N.; Thai, T.H.; Huyen, D.D.; Thang, T.D.; Ogunwande, I.A. The leaf essential oils of four Vietnamese species of Cinnamomum (Lauraceae). J. Essent. Oil Res. 2013, 25, 267-271. [CrossRef]

33. Son, L.C.; Dai, D.N.; Thang, T.D.; Huyen, D.D.; Olayiwola, T.O.; Ogunmoye, A.R.; Ogunwande, I.A. Chemical composition of essential oils from the stem barks of three Cinnamomum species. Br. J. Appl. Sci. Technol. 2015, 11, 20442. [CrossRef]

34. Son, L.C.; Dai, D.N.; Thang, T.D.; Huyen, D.D.; Ogunwande, I.A. Study on Cinnamomum oils: Compositional pattern of seven species grown in Vietnam. J. Oleo Sci. 2014, 63, 1035-1043. [CrossRef]

35. Dorman, H.J.D.; Deans, S.G. Antimicrobial agents from plants: Antibacterial activity of plant volatile oils. J. Appl. Microbiol. 2000, 88, 308-316. [CrossRef]

36. Gallucci, M.N.; Oliva, M.; Casero, C.; Dambolena, J.; Luna, A.; Zygadlo, J.; Demo, M. Antimicrobial combined action of terpenes against the food-borne microorganisms Escherichia coli, Staphylococcus aureus and Bacillus cereus. Flavour Fragr. J. 2009, 24, 348-354. [CrossRef]

37. Boonchird, C.; Flegel, T.W. In vitro antifungal activity of eugenol and vanillin against Candida albicans and Cryptococcus neoformans. Can. J. Microbiol. 1982, 28, 1235-1241. [CrossRef]

38. Cheng, S.-S.; Liu, J.-Y.; Chang, E.-H.; Chang, S.-T. Antifungal activity of cinnamaldehyde and eugenol congeners against wood-rot fungi. Bioresour. Technol. 2008, 99, 5145-5149. [CrossRef] [PubMed]

39. Abbaszadeh, S.; Sharifzadeh, A.; Shokri, H.; Khosravi, A.R.; Abbaszadeh, A. Antifungal efficacy of thymol, carvacrol, eugenol and menthol as alternative agents to control the growth of food-relevant fungi. J. Mycol. Med. 2014, 24, 51-56. [CrossRef] [PubMed]

40. Cheng, S.-S.; Liu, J.-Y.; Tsai, K.-H.; Chen, W.-J.; Chang, S.-T. Chemical composition and mosquito larvicidal activity of essential oils from leaves of different Cinnamomum osmophloeum provenances. J. Agric. Food Chem. 2004, 52, 4395-4400. [CrossRef]

41. Govindarajan, M.; Rajeswary, M.; Hoti, S.L.; Bhattacharyya, A.; Benelli, G. Eugenol, $\alpha$-pinene and $\beta$-caryophyllene from Plectranthus barbatus essential oil as eco-friendly larvicides against malaria, dengue and Japanese encephalitis mosquito vectors. Parasitol. Res. 2016, 115, 807-815. [CrossRef]

42. Pavela, R. Insecticidal properties of phenols on Culex quinquefasciatus Say and Musca domestica L. Parasitol. Res. 2011, 109, 1547-1553. [CrossRef] 
43. Schmidt, J.M.; Noletto, J.A.; Vogler, B.; Setzer, W.N. Abaco bush medicine: Chemical composition of the essential oils of four aromatic medicinal plants from Abaco Island, Bahamas. J. Herbs. Spices Med. Plants 2006, 12, 43-65. [CrossRef]

44. Wanner, J.; Schmidt, E.; Bail, S.; Jirovetz, L.; Buchbauer, G.; Gochev, V.; Girova, T.; Atanasova, T.; Stoyanova, A. Chemical composition and antibacterial activity of selected essential oils and some of their main compounds. Nat. Prod. Commun. 2010, 5, 1359-1364. [CrossRef]

45. Filipowicz, N.; Kaminski, M.; Kurlenda, J.; Asztemborska, M.; Ochocka, J.R. Antibacterial and antifungal activity of juniper berry oil and its selected components. Phyther. Res. 2003, 17, 227-231. [CrossRef]

46. Hammer, K.A.; Carson, C.F.; Riley, T. V Antifungal activity of the components of Melaleuca alternifolia (tea tree) oil. J. Appl. Microbiol. 2003, 95, 853-860. [CrossRef] [PubMed]

47. Jantan, I.; Moharam, B.A.K.; Santhanam, J.; Jamal, J.A. Correlation between chemical composition and antifungal activity of the essential oils of eight Cinnamomum species. Pharm. Biol. 2008, 46, 406-412. [CrossRef]

48. Hung, N.H.; Satyal, P.; Hieu, H.V.; Chuong, N.T.H.; Dai, D.N.; Huong, L.T.; Tai, T.A.; Setzer, W.N. Mosquito larvicidal activity of the essential oils of Erechtites species growing wild in Vietnam. Insects 2019, 10, 47. [CrossRef] [PubMed]

49. Govindarajan, M. Chemical composition and larvicidal activity of leaf essential oil from Clausena anisata (Willd.) Hook. f. ex Benth (Rutaceae) against three mosquito species. Asian Pac. J. Trop. Med. 2010, 3, 874-877. [CrossRef]

50. Cheng, S.-S.; Huang, C.-G.; Chen, Y.-J.; Yu, J.-J.; Chen, W.-J.; Chang, S.-T. Chemical compositions and larvicidal activities of leaf essential oils from two eucalyptus species. Bioresour. Technol. 2009, 100, 452-456. [CrossRef]

51. Evergetis, E.; Michaelakis, A.; Haroutounian, S.A. Exploitation of Apiaceae family essential oils as potent biopesticides and rich source of phellandrenes. Ind. Crops Prod. 2013, 41, 365-370. [CrossRef]

52. bin Jantan, I.; Yalvema, M.F.; Ahmad, N.W.; Jamal, J.A. Insecticidal activities of the leaf oils of eight Cinnamomum species against Aedes aegypti and Aedes albopictus. Pharm. Biol. 2005, 43, 526-532. [CrossRef]

53. de Morais, S.M.; Facundo, V.A.; Bertini, L.M.; Cavalcanti, E.S.B.; dos Anjos Júnior, J.F.; Ferreira, S.A.; de Brito, E.S.; de S Neto, M.A. Chemical composition and larvicidal activity of essential oils from Piper species. Biochem. Syst. Ecol. 2007, 35, 670-675. [CrossRef]

54. Pattnaik, S.; Subramanyam, V.R.; Bapaji, M.; Kole, C.R. Antibacterial and antifungal activity of aromatic constituents of essential oils. Microbios 1997, 89, 39-46.

55. Setzer, W.N.; Vogler, B.; Schmidt, J.M.; Leahy, J.G.; Rives, R. Antimicrobial activity of Artemisia douglasiana leaf essential oil. Fitoterapia 2004, 75. [CrossRef]

56. Satyal, P.; Paudel, P.; Poudel, A.; Dosoky, N.S.; Pokharel, K.K.; Setzer, W.N. Bioactivities and compositional analyses of Cinnamomum essential oils from Nepal: C. camphora, C. tamala and C. glaucescens. Nat. Prod. Commun. 2013, 8, 1777-1784. [CrossRef] [PubMed]

57. Setzer, W.N.; Schmidt, J.M.; Eiter, L.C.; Haber, W.A. The leaf oil composition of Zanthoxylum fagara (L.) Sarg. from Monteverde, Costa Rica and its biological activities. J. Essent. Oil Res. 2005, 17, 333-335. [CrossRef]

58. Reichling, J.; Suschke, U.; Schneele, J.; Geiss, H.K. Antibacterial activity and irritation potential of selected essential oil components-Structure-activity relationship. Nat. Prod. Commun. 2006, 1, 1003-1012. [CrossRef]

59. Fisher, K.; Phillips, C.A. The effect of lemon, orange and bergamot essential oils and their components on the survival of Campylobacter jejuni, Escherichia coli O157, Listeria monocytogenes, Bacillus cereus and Staphylococcus aureus in vitro and in food systems. J. Appl. Microbiol. 2006, 101, 1232-1240. [CrossRef]

60. Mesa-Arango, A.C.; Montiel-Ramos, J.; Zapata, B.; Durán, C.; Betancur-Galvis, L.; Stashenko, E. Citral and carvone chemotypes from the essential oils of Colombian Lippia alba (Mill.) N.E. Brown: Composition, cytotoxicity and antifungal activity. Mem. Inst. Oswaldo Cruz 2009, 104, 878-884. [CrossRef]

61. Silva, C.D.B.D.; Guterres, S.S.; Weisheimer, V.; Schapoval, E.E. Antifungal activity of the lemongrass oil and citral against Candida spp. Braz. J. Infect. Dis. 2008, 12, 63-66. [CrossRef]

62. Cheng, S.-S.; Liu, J.-Y.; Huang, C.-G.; Hsui, Y.-R.; Chen, W.-J.; Chang, S.-T. Insecticidal activities of leaf essential oils from Cinnamomum osmophloeum against three mosquito species. Bioresour. Technol. 2009, 100, 457-464. [CrossRef] 
63. Radwan, M.A.; El-Zemity, S.R.; Mohamed, S.A.; Sherby, S.M. Larvicidal activity of some essential oils, monoterpenoids and their corresponding $N$-methyl carbamate derivatives against Culex pipiens (Diptera: Culicidae). Int. J. Trop. Insect Sci. 2008, 28, 61-68. [CrossRef]

64. Adams, R.P. Identification of Essential Oil Components by Gas Chromatography/Mass Spectrometry, 4th ed.; Allured Publishing: Carol Stream, IL, USA, 2007.

65. Sahm, D.H.; Washington, J.A. Antibacterial susceptibility tests: Dilution methods. In Manual of Clinical Microbiology; Balows, A., Hausler, W.J., Herrmann, K.L., Isenberg, H.D., Shamody, H.J., Eds.; American Society for Microbiology: Washington, DC, USA, 1991.

66. Liu, Z.L.; He, Q.; Chu, S.S.; Wang, C.F.; Du, S.S.; Deng, Z.W. Essential oil composition and larvicidal activity of Saussurea lappa roots against the mosquito Aedes albopictus (Diptera: Culicidae). Parasitol. Res. 2012, 110, 2125-2130. [CrossRef]

67. Finney, D. Probit Analysis; Vincent, K., Ed.; Cambridge University Press: Cambridge, UK, 2009; ISBN 978-0521135900.

68. Duarte, M.C.T.; Leme, E.E.; Delarmelina, C.; Soares, A.A.; Figueira, G.M.; Sartoratto, A. Activity of essential oils from Brazilian medicinal plants on Escherichia coli. J. Ethnopharmacol. 2007, 111, 197-201. [CrossRef]

69. Dias, C.N.; Moraes, D.F.C. Essential oils and their compounds as Aedes aegypti L. (Diptera: Culicidae) larvicide: Review. Parasitol. Res. 2014, 113, 565-592. [CrossRef] [PubMed]

Sample Availability: The Cinnamomum essential oils are no longer available. 